\title{
Solving the Classical Nucleation Theory with respect to the surface energy
}

\author{
Daniel R. Cassar \\ Department of Materials Engineering, Federal University of São Carlos, São Carlos, Brazil
}

\begin{abstract}
An essential parameter of the Classical Nucleation Theory (CNT) is the surface energy between a critical-size nucleus and the ambient phase, $\sigma$. In condensed matter, this parameter cannot be experimentally determined independently of CNT. A common practice to obtain $\sigma$ is to assume a model for its temperature-dependence and perform a regression of the CNT equation against experimental nucleation data. The drawback of this practice is that assuming the temperaturedependence of $\sigma$ adds a bias to the analysis. Nonetheless, this practice is common because an analytical solution of the Classical Nucleation Theory with respect to $\sigma$ is not possible considering common expressions of this theory. In this article, a general numerical solution to this problem using the Lambert $W$ function is proposed, tested, and compared with typical regression methods. The major advantage of the proposed method is that there is no need to assume a model for the temperature-dependence of $\sigma$.
\end{abstract}

Keywords: Classical Nucleation Theory, surface energy, Lambert $W$ function

\section{Classical Nucleation Theory}

The Classical Nucleation Theory (CNT) formulation shown in Eq. (1) is based on the work of Gibbs [1] with further modifications from other authors [2-6]. In this equation, $J$ is the nucleation rate, $J_{0}$ is a pre-exponential factor, $D$ is the effective diffusion coefficient related to the mobility of the units that participate in the nucleation process, $Z$ is the Zeldovich factor [5], $W^{*}$ is the work of formation of a critical-size nucleus, $k$ is the Boltzmann constant, and $T$ is the absolute temperature.

$$
J=J_{0} D Z \exp \left(-\frac{W^{*}}{k T}\right)
$$

Some expressions exist for the parameters $J_{0}, D, Z$, and $W^{*}$ [7]. These expressions depend on specific considerations about the nucleation process. For instance, if the critical-size nucleus is considered to be spherical and isotropic, then

$$
W^{*}=\frac{16 \pi \sigma^{3}}{3 \Delta G_{V}^{2}},
$$

where $\sigma$ is the surface energy between the nucleus and the ambient phase, and $\Delta G_{V}$ is the change in the Gibbs free energy (per unit of volume) when the ambient phase transforms into the phase of the nucleus $[8,9]$.

(C) 2019. This manuscript version is made available under the CC-BY-NC-ND 4.0 license http://creativecommons.org/licenses/bync-nd $/ 4.0 /$

* Corresponding author

Email address: daniel.r.cassar@gmail.com (Daniel R. Cassar)
Eqs. (2) and (3) show two expressions for Eq. (1) found in the literature $[7,8,10-12]$, considering a plethora of assumptions discussed therein.

$$
\begin{gathered}
J=\frac{\sqrt{k T \sigma}}{\eta \lambda^{5}} \exp \left(-\frac{16 \pi \sigma^{3}}{3 k T \Delta G_{V}^{2}}\right) \\
J=\frac{16 \sqrt{k T \sigma^{3}}}{3 \tau \Delta G_{V}^{2} \lambda^{6}} \exp \left(-\frac{16 \pi \sigma^{3}}{3 k T \Delta G_{V}^{2}}\right)
\end{gathered}
$$

In the previous expressions, $\eta$ is the shear viscosity of the ambient phase, $\lambda$ is the jump distance for diffusion estimated by the size of the units that diffuse during the nucleation process, and $\tau$ is the time-lag of the nucleation process.

Eqs. (2) and (3) cannot be solved analytically for $\sigma$. The objective of this short communication is to show how to solve this kind of problem numerically using the Lambert $W$ function [13].

The domain of Eqs. (2) and (3) is dependent on the assumptions used to obtain them; here they will be used only for experimental nucleation rate data above the reported temperature of maximum nucleation. Fortunately, the method proposed here is general and can also be used for other expressions of $J$.

\section{Lambert $W$ function}

The Lambert $W$ function (also known as the omega function) is defined as the inverse function of $f(z)=z \exp (z)$, with $z$ being any complex number, thus

$$
z=W_{n}(z \exp (z)) .
$$




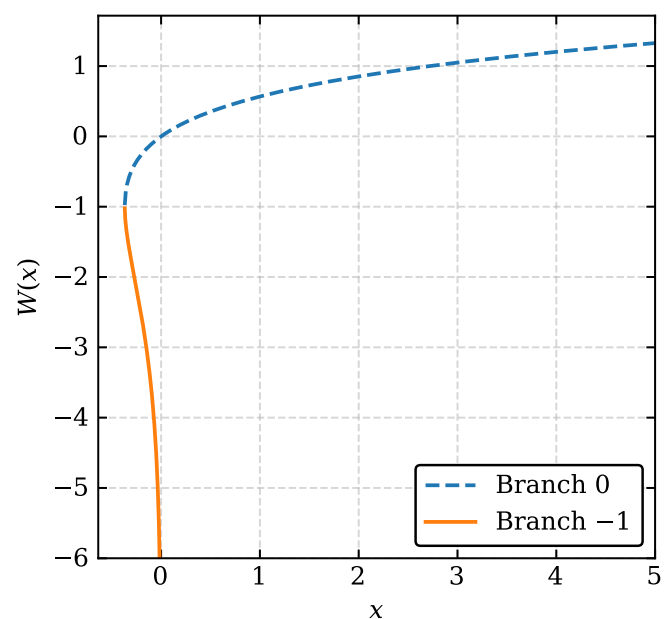

Figure 1: Real values of the $W$ functions computed in the branches 0 and -1 .

Table 1: Parameters $a, p, b$, and $q$ for Eqs. (2) and (3). The parameters $b$ and $q$ are the same for both equations.

\begin{tabular}{ccccc}
\hline & $a$ & $p$ & $b$ & $q$ \\
\hline \hline $\mathrm{Eq} .(2)$ & $\sqrt{k T}\left[J \eta \lambda^{5}\right]^{-1}$ & $1 / 2$ & $-\frac{16 \pi}{3 \Delta G_{V}^{2} k T}$ & 3 \\
$\mathrm{Eq} .(3)$ & $16 \sqrt{k T}\left[3 J \Delta G_{V}^{2} \tau \lambda^{6}\right]^{-1}$ & $3 / 2$ & & \\
\hline
\end{tabular}

The $W$ is a multivalued function with $n$ branches, and one must select an integer $n$ to solve it. Only $n=0$ and -1 produce real results, the only ones of interest to the problem here, considering there is no physical meaning for complex values of $\sigma$. Figure 1 shows the real values of $W$ for these two branches.

Eqs. (2) and (3) can be written in the form

$$
a \sigma^{p} \exp \left(b \sigma^{q}\right)=1,
$$

where $a, p, b$, and $q$ are non-zero parameters that depend on the nucleation expression in consideration (see Table 1). A solution of Eq. (4) for $\sigma$ is

$$
\sigma=\left[\frac{p}{b q} W_{n}\left(\frac{b q}{p} a^{-\frac{q}{p}}\right)\right]^{\frac{1}{q}} .
$$

With the solution shown in (5), it is possible to solve expressions (2) and (3) for $\sigma$. In fact, any equation that can be written in the form of (4) can be solved for $\sigma$. This solution may also be used, for example, when dealing with crystal growth mediated by secondary surface nucleation (see Ref. [14]). The fact that a solution is possible does not imply that it is non-complex. In some cases, a real solution may be impossible.

Considering that $\sigma$ is expected to increase monotonically with respect to the temperature in the range where nucleation data are available [15], the only physically valid branch of $W$ in this scenario is branch -1 .

Several free and commercial numerical programs have an implementation of the $W$ function. Scipy [16], for in-
Table 2: Crystal nucleation data and properties of interest of $\mathrm{Li}_{2} \mathrm{~B}_{4} \mathrm{O}_{7}$ [18]. Uncertainty in $J, \tau$, and $\eta$ is one standard deviation. Uncertainty in $J$ and $\tau$ is the standard deviation obtained in the parameters of the non-linear regression of the Collins-Kashchiev equation [23, 24] (see [18] for more details). Uncertainty in $\eta$ is the confidence band of the shear viscosity regression.

\begin{tabular}{ccccc}
\hline$T$ & $J \times 10^{-7}$ & $\tau$ & $\Delta G_{V} \times 10^{-8}$ & $\eta \times 10^{-9}$ \\
{$[\mathrm{~K}]$} & {$\left[\mathrm{m}^{-3} \mathrm{~s}^{-1}\right]$} & {$[\mathrm{s}]$} & {$\left[\mathrm{J} \mathrm{m}^{-3}\right]$} & {$[\mathrm{Pas}]$} \\
\hline \hline 773 & $90(1)$ & $1323(8)$ & -4.20 & $54(2)$ \\
783 & $64(1)$ & $877(9)$ & -4.15 & $7.0(2)$ \\
793 & $6.9(1)$ & $140(10)$ & -4.10 & $1.10(4)$ \\
\hline
\end{tabular}

Table 3: Values of $\sigma$ computed using data from Table 2, considering $\lambda=4.86 \times 10^{-10} \mathrm{~m}$ and uncertainty of $2 \mathrm{~K}$ in $T$. $T$ in $\mathrm{K}$ and $\sigma$ in $\mathrm{J} \mathrm{m}^{-2}$. Values obtained using branch -1 of the Lambert $W$ function.

\begin{tabular}{ccc}
\hline & Eq. (2) & Eq. (3) \\
$T$ & $\sigma$ & $\sigma$ \\
\hline \hline 773 & $0.1621(2)$ & $0.1608(1)$ \\
783 & $0.1649(2)$ & $0.1614(2)$ \\
793 & $0.1697(2)$ & $0.1664(2)$ \\
\hline
\end{tabular}

stance, is a free and open-source module for the Python programming language that has this function built in with the name lambertW, available in the scipy.special submodule. For an iterative approach that can be performed using a spreadsheet program, see Ref. [17].

\section{Test on experimental data}

Table 2 shows crystal nucleation data measured in a supercooled $\mathrm{Li}_{2} \mathrm{~B}_{4} \mathrm{O}_{7}$ liquid (lithium tetraborate, also known as lithium diborate), as well as other properties of interest [18]. With these data and the solution shown in Eq. (5), the value of $\sigma$ was computed considering the two expressions for $J$ that are common in the literature (Eqs. (2) and (3)). The results are shown in Table 3. The computed $\sigma$ values are within the expected range for this parameter (0.1 to $\left.0.25 \mathrm{~J} \mathrm{~m}^{-2}\right)$ [10, 19-22].

\section{Comparison with regression methods}

Another approach to obtain $\sigma$ from experimental $J(T)$ data is via regression. A method popularized by James [25] consists of linearizing Eq. (2) by plotting $\ln \left(J \eta T^{-1 / 2}\right)$ versus $\left[T \Delta G_{V}^{2}\right]^{-1}$, with the underlying assumption that $\sigma$ and $\lambda$ are temperature-independent. With a linear regression of the data in the linearized plot, $\sigma$ can be obtained from the slope of the regression, which is equal to $-16 \pi \sigma^{3}[3 k]^{-1}$, and $\lambda$ can be obtained from the intercept of the regression, which is equal to $\ln \left(\sqrt{k T \sigma} \lambda^{-5}\right)$.

Values of $\sigma=0.32(1) \mathrm{J} \mathrm{m}^{-2}$ and $\lambda=1(5) \times 10^{-32} \mathrm{~m}$ were obtained using this method with the data shown in 
Table 4: Reported values of $J$ and calculated values of $J$ using different methods. See the text for a description of each method. $T$ in $\mathrm{K}$ and $J$ in $\mathrm{m}^{-3} \mathrm{~s}^{-1}$

\begin{tabular}{ccccc}
\hline & \multicolumn{4}{c}{$J \times 10^{-7}$} \\
\cline { 2 - 5 }$T$ & Reported & Method 1 & Method 2 & Method 3 \\
\hline \hline 773 & $90(1)$ & 110 & 90 & 90 \\
783 & $64(1)$ & 45 & 64 & 64 \\
793 & $6.9(1)$ & 8.1 & 31 & 6.9 \\
\hline
\end{tabular}

Table 2. The value of $\sigma$ is two times higher than the value obtained using the Lambert $W$ function. The value of $\lambda$ is 22 orders of magnitude smaller than what is expected, which is a discrepancy that is well documented in the literature $[8,10]$. Rarely discussed, however, is the uncertainty in $\lambda$ when using this method, which is massive for the dataset investigated here.

As a consistency test, Table 4 shows the computed value of $J$ when putting the values of $\sigma$ and $\lambda$ obtained using this method back into Eq. (2). The computed value is shown in the column "Method 1". The difference between the reported and the computed value is up to $42 \%$ for the temperature of $783 \mathrm{~K}$.

A similar approach using Eq. (3) instead of Eq. (2) was proposed by Weinberg and Zanotto [26], which yields similar results (not shown here). One advantage of these two regression approaches (advanced by James, Weinberg, and Zanotto) is that no assumption must be made on the value of $\lambda$. However, the resulting uncertainty in $\lambda$ is enough to discard this apparent advantage in this case. Moreover, assuming $\sigma$ to be temperature-independent may be a reasonable approximation only for sufficiently small temperature ranges.

Another regression approach is to assume some model for the temperature-dependence of $\sigma$ and use it to perform a non-linear regression of CNT. The expression $\sigma=$ $2.8 \times 10^{-4} T-0.054$ is obtained if we consider Eq. (2), $\lambda=4.86 \times 10^{-10} \mathrm{~m}$, and assume that $\sigma$ has a linear temperature-dependence. The computed values of $J$ using this method are shown in the column "Method 2" in Table 4. The difference between the reported and the computed value is $78 \%$ for the temperature of $793 \mathrm{~K}$.

Finally, Table 4 also shows the calculated values of $J$ using the method proposed in this article, column "Method 3 ". It is no surprise that these values are equal to the experimental values because no assumption concerning the temperature-dependence of $\sigma$ is necessary to use this new method; thus it gives the exact value of $\sigma$ to bring theory and experiment to an agreement, point by point. The hope is that with fewer assumptions we can reach new insights into this problem.

\section{Conclusion}

An analytical solution for the surface energy may not be possible depending on the form of Classical Nucleation
Theory that is used. Here I demonstrate a numerical solution (using the Lambert $W$ function) to solve the Classical Nucleation Theory with respect to the surface energy. The advantage of this numerical approach, in contrast with previous methods, is that no assumption on the temperaturedependence of the surface energy is necessary.

\section{Acknowledgements}

Daniel R. Cassar gratefully acknowledges the São Paulo Research Foundation for the financial support (FAPESP grant number 2017/12491-0) and Carolina B. Zanelli for the English revision.

\section{References}

[1] J. W. Gibbs, On the equilibrium of heterogeneous substances, Transactions of the Connecticut Academy of Arts and Sciences 3 (1876) 108-248 and 343-524 (1876).

[2] M. Volmer, A. Weber, Nucleation in super-saturated products, Zeitschrift für Physikalische Chemie 119 (1926) 277-301 (1926).

[3] L. Farkas, Keimbildungsgeschwindigkeit in übersättigten Dämpfen, Zeitschrift für physikalische Chemie 125 (1) (1927) 236-242 (1927)

[4] R. Kaischew, I. N. Stranski, Zur kinetischen Ableitung der Keimbildungsgeschwindigkeit, Zeitschrift für Physikalische Chemie 26 (1934) 317-326 (1934).

[5] Y. B. Zeldovich, Contribution to the theory of the formation of a new phase, Zhurnal Eksperimentalnoi i Teoreticheskoi Fiziki 12 (1942) 525-538 (1942).

[6] J. Frenkel, Kinetic Theory of Liquids, Oxford University Press, Oxford, 1946 (1946).

[7] J. W. P. Schmelzer, G. Röpke, V. Priezzhev, Nucleation theory and applications, Wiley Online Library, Weinheim, 2005 (2005). doi:10.1002/3527604790. fmatter/pdf.

[8] K. F. Kelton, Crystal nucleation in liquids and glasses, in: H. Ehrenreich, D. Turnbull (Eds.), Solid State Physics, Vol. Volume 45, Academic Press, Berlin, 1991, pp. 75-177 (1991).

[9] D. R. Cassar, Crystallization driving force of supercooled oxide liquids, International Journal of Applied Glass Science 7 (3) (2016) 262-269 (2016). doi:10.1111/ijag.12218.

[10] V. M. Fokin, E. D. Zanotto, N. S. Yuritsyn, J. W. P. Schmelzer, Homogeneous crystal nucleation in silicate glasses: A 40 years perspective, Journal of Non-Crystalline Solids 352 (26-27) (2006) 2681-2714 (2006). doi:10.1016/j.jnoncrysol.2006. 02.074 .

[11] P. K. Gupta, D. R. Cassar, E. D. Zanotto, Role of dynamic heterogeneities in crystal nucleation kinetics in an oxide supercooled liquid, The Journal of Chemical Physics 145 (21) (2016) 211920 (2016). doi:10.1063/1.4964674.

[12] P. K. Gupta, D. R. Cassar, E. D. Zanotto, On the variation of the maximum crystal nucleation rate temperature with glass transition temperature, Journal of Non-Crystalline Solids 442 (2016) 34-39 (2016). doi:10.1016/j . jnoncrysol.2016.03.024.

[13] R. M. Corless, G. H. Gonnet, D. E. G. Hare, D. J. Jeffrey, D. E. Knuth, On the LambertW function, Advances in Computational Mathematics 5 (1) (1996) 329-359 (1996). doi: 10.1007/BF02124750.

[14] D. R. Cassar, R. F. Lancelotti, R. Nuernberg, M. L. F. Nascimento, A. M. Rodrigues, L. T. Diz, E. D. Zanotto, Elemental and cooperative diffusion in a liquid, supercooled liquid and glass resolved, The Journal of Chemical Physics 147 (1) (2017) 014501 (2017). doi:10.1063/1.4986507.

[15] A. L. Greer, K. F. Kelton, Nucleation in Lithium Disilicate Glass: A Test of Classical Theory by Quantitative Modeling, Journal of the American Ceramic Society 74 (5) (1991) 10151022 (1991). doi:10.1111/j.1151-2916.1991.tb04337.x. 
[16] E. Jones, T. Oliphant, P. Peterson, others, SciPy: Open source scientific tools for Python, 2001 (2001).

[17] D. C. Allan, Inverting the MYEGA equation for viscosity, Journal of Non-Crystalline Solids 358 (2) (2012) 440-442 (2012). doi:10.1016/j.jnoncrysol.2011.09.036.

[18] D. R. Cassar, Crystal nucleation, growth, relaxation and viscous flow in diopside and lithium diborate glasses, Ph.D. thesis, Universidade Federal de São Carlos, São Carlos (2014).

[19] J. Deubener, M. Weinberg, Crystal-liquid surface energies from transient nucleation, Journal of Non-Crystalline Solids 231 (1-2) (1998) 143-151 (1998). doi:10.1016/S0022-3093(98) 00412-8.

[20] V. M. Fokin, E. D. Zanotto, Crystal nucleation in silicate glasses: the temperature and size dependence of crystal/liquid surface energy, Journal of Non-Crystalline Solids $265(1-2)$ (2000) 105-112 (2000). doi:10.1016/S0022-3093(99) 00877-7.

[21] V. M. Fokin, E. D. Zanotto, J. W. P. Schmelzer, Method to estimate crystal/liquid surface energy by dissolution of subcritical nuclei, Journal of Non-Crystalline Solids 278 (1-3) (2000) 24-34 (2000). doi:10.1016/S0022-3093(00)00335-5.

[22] J. W. P. Schmelzer, A. S. Abyzov, E. B. Ferreira, V. M. Fokin, Curvature dependence of the surface tension and crystal nucleation in liquids, International Journal of Applied Glass Science 10 (1) (2019) 57-68 (2019). doi:10.1111/ijag.12900.

[23] F. C. Collins, Time Lag in Spontaneous Nucleation Due to NonSteady State Effects, Zeitschrift für Elektrochemie, Berichte der Bunsengesellschaft für physikalische Chemie 59 (5) (1955) 404407 (1955). doi:10.1002/bbpc. 19550590515.

[24] D. Kashchiev, Solution of the non-steady state problem in nucleation kinetics, Surface Science 14 (1) (1969) 209-220 (1969). doi: 10.1016/0039-6028(69) 90055-7.

[25] P. F. James, Kinetics of crystal nucleation in silicate glasses, Journal of Non-Crystalline Solids 73 (1-3) (1985) 517-540 (1985). doi : 10.1016/0022-3093(85) 90372-2.

[26] M. C. Weinberg, E. D. Zanotto, Re-examination of the temperature dependence of the classical nucleation rate: homogeneous crystal nucleation in glass, Journal of non-crystalline solids 108 (1989) 99-108 (1989). 